“ (C) 2013 IEEE. Personal use of this material is permitted. Permission from IEEE must be obtained for all other uses, in any current or future media, including

reprinting/republishing this material for advertising or promotional purposes, creating new collective works, for resale or redistribution to servers or lists, or reuse of any copyrighted component of this work in other works." 


\title{
A Tight Upper Bound for the Third-Order Asymptotics for Most Discrete Memoryless Channels
}

\author{
Marco Tomamichel, Member, IEEE and Vincent Y. F. Tan, Member, IEEE
}

\begin{abstract}
This paper shows that the logarithm of the $\varepsilon$-error capacity (average error probability) for $n$ uses of a discrete memoryless channel (DMC) is upper bounded by the normal approximation plus a third-order term that does not exceed $\frac{1}{2} \log n+O(1)$ if the $\varepsilon$-dispersion of the channel is positive. This matches a lower bound by Y. Polyanskiy (2010) for DMCs with positive reverse dispersion. If the $\varepsilon$-dispersion vanishes, the logarithm of the $\varepsilon$-error capacity is upper bounded by $n$ times the capacity plus a constant term except for a small class of DMCs and $\varepsilon \geq \frac{1}{2}$.
\end{abstract}

\section{INTRODUCTION}

The primary information-theoretic task in point-to-point channel coding is the characterization of the maximum rate of communication over $n$ independent uses of a noisy channel $W$. We are concerned in this paper with discrete memoryless channels (DMCs). Let $M^{*}\left(W^{n}, \varepsilon\right)$ resp. $M_{\max }^{*}\left(W^{n}, \varepsilon\right)$ denote the maximum size of a length- $n$ block code for DMC $W$ having average resp. maximal error probability no larger than $\varepsilon \in(0,1)$. Shannon's noisy-channel coding theorem [1] and Wolfowitz's strong converse [2] state that for every $\varepsilon \in(0,1)$,

$$
\lim _{n \rightarrow \infty} \frac{1}{n} \log M^{*}\left(W^{n}, \varepsilon\right)=C \quad \text { bits/channel use, }
$$

where $C:=\max _{P} I(P, W)$ is the channel capacity. Since the 1960 s, there has been interest in determining finer asymptotic characterizations of the coding theorem. This is useful because such an analysis provides key insights into the amount of backoff from channel capacity for block codes of finite length $n$. In particular, Strassen in 1962 [3] showed using normal approximations that the asymptotic expansion of $\log M_{\max }^{*}\left(W^{n}, \varepsilon\right)$ satisfies

$$
\log M_{\max }^{*}\left(W^{n}, \varepsilon\right)=n C+\sqrt{n V_{\varepsilon}} \Phi^{-1}(\varepsilon)+\rho_{n},
$$

where $\rho_{n}=O(\log n), V_{\varepsilon}$ is the $\varepsilon$-channel dispersion [4], [5] and $\Phi(\cdot)$ is the Gaussian cumulative distribution function 1 These quantities will be defined precisely in Section $\amalg-\mathrm{A}$ In fact, this asymptotic expansion also holds for $M^{*}\left(W^{n}, \varepsilon\right)$ [4, Eqs. (284)-(286)] and implies that if an error probability of $\varepsilon$ is tolerable, the backoff from channel capacity $C$ at finite blocklength $n$ is roughly $\sqrt{V_{\varepsilon} / n} \Phi^{-1}(\varepsilon)$. There have been several recent refinements to and extensions of Strassen's normal approximation in (1), most prominently by Hayashi [6] and Polyanskiy et al. [4]. Strassen's normal approximation has also been shown to hold for many other classes of channels such as the additive white Gaussian noise (AWGN) channel [4]-[6] and the additive Markovian channel [6].

M. Tomamichel is with the Centre of Quantum Technologies, National University of Singapore, 3 Science Drive 2, Singapore 117542. (email: cqtmarco@nus.edu.sg). V. Y. F. Tan is with the Institute for Infocomm Research $\left(\mathrm{I}^{2} \mathrm{R}\right)$, A*STAR, Singapore and with the Department of Electrical and Computer Engineering, National University of Singapore. (email: vtan@ nus.edu.sg). This paper was presented in part at the IEEE International Symposium on Information Theory (ISIT 2013).

${ }^{1}$ In fact, it was pointed out by Polyanskiy [5] Sec. 3.4.1] that Strassen's paper [3, Thm. 1.2] contains a gap in the case when the DMC is exotic and $\varepsilon>\frac{1}{2}$. 
Despite these impressive advances in the fundamental limits of channel coding, the third-order term $\rho_{n}$ is not well understood. Indeed, Hayashi in the conclusion of his paper [6] mentions that

"... the third-order coding rate is expected but appears difficult. The second order is the order $\sqrt{n}$, and it is not clear whether the third-order is a constant order or the order $\log n$ "

What we do know is that for the binary symmetric channel (BSC), $\rho_{n}=\frac{1}{2} \log n+O(1)$ [4] Thm. 52] and for the binary erasure channel (BEC), $\rho_{n}=O(1)$ [4, Thm. 53]. More generally, there are classes of channels for which we have bounds on $\rho_{n}$ [5, Sec. 3.4.5]. For lower bounds (achievability), if we consider DMCs $W$ with positive reverse dispersion [5, Eq. (3.296)], then $\rho_{n} \geq \frac{1}{2} \log n+O(1)$ [5. Cor. 54]. For upper bounds (converse), if we restrict our attention to so-called weakly inputsymmetric DMCs [5], Def. 9], $\rho_{n} \leq \frac{1}{2} \log n+O(1)$ [5, Thm. 55]. For constant-composition codes, it was shown [7] using strong large-deviation techniques [8], [9] that, under some regularity assumptions, $\rho_{n}=\frac{1}{2} \log n+O(1)$. Recall that a constant-composition code is one where all the codewords are of the same empirical distribution or type. It is also claimed that the same holds for a more general class of DMCs in [10]. Our results generalize the converse bounds in [7] and [10].

This paper strengthens the upper bound (converse) on the third-order term $\rho_{n}$. For all DMCs whose $\varepsilon$-dispersions are positive, we show that

$$
\log M^{*}\left(W^{n}, \varepsilon\right) \leq n C+\sqrt{n V_{\varepsilon}} \Phi^{-1}(\varepsilon)+\frac{1}{2} \log n+O(1),
$$

If the $\varepsilon$-dispersion vanishes, the corresponding bound is $\log M^{*}\left(W^{n}, \varepsilon\right) \leq n C+O(1)$, unless the DMC is exotic [4. Thm. 48] and $\varepsilon \geq \frac{1}{2}$. If the DMC is exotic and $\varepsilon=\frac{1}{2}$, we show that $\log M^{*}\left(W^{n}, \frac{1}{2}\right) \leq$ $n C+\frac{1}{2} \log n+O(1)$. If the DMC is exotic and $\varepsilon>\frac{1}{2}, \log M^{*}\left(W^{n}, \varepsilon\right) \leq n C+O\left(n^{\frac{1}{3}}\right)$, a result by Polyanskiy et al. [4, Thm. 48]. Hence, for the rather general class of DMCs with positive $\varepsilon$-dispersion, the third-order term is $\rho_{n} \leq \frac{1}{2} \log n+O(1)$. We may thus dispense with the assumption that $W$ is weakly input-symmetric [5, Def. 9].

The typical way [3]-[5] to upper bound $M^{*}\left(W^{n}, \varepsilon\right)$ is to first do the same for the maximum size of a constant-composition code under the maximum error probability formulation. Such a bound can be proved using either the meta-converse [4, Thm. 31] or tight bounds on the type-II error probability in a simple binary hypothesis test [3, Thm. 1.1]. By the type-counting lemma [11, Lem. 2.2], every length- $n$ block code can be partitioned into no more than $(n+1)^{|\mathcal{X}|-1}$ constant-composition subcodes. This leads to the rather conservative bound [3, Eq. (4.29)] [4, Eq. (279)]

$$
\log M_{\max }^{*}\left(W^{n}, \varepsilon\right) \leq n C+\sqrt{n V_{\varepsilon}} \Phi^{-1}(\varepsilon)+\left(|\mathcal{X}|-\frac{1}{2}\right) \log n+O(1) .
$$

Subsequently, by expurgating bad codewords (see [4, Eqs. (284)-(286)]), we can conclude that the same upper bound holds for $M^{*}\left(W^{n}, \varepsilon\right)$. We adopt a different approach for the proof of our main result in (2) and work with $M^{*}\left(W^{n}, \varepsilon\right)$ directly. In a nutshell, we consider a new "symbol-wise" relaxation of the meta-converse that allows us to work directly with general (non-constant-composition) codes and the average probability of error. The one-shot converse is stated in terms of the relative entropy information spectrum [12, Ch. 4] but allows us to choose an auxiliary output distribution as in the meta-converse. We then carefully weigh the contributions of each input type for a general code by constructing an appropriate $\epsilon$-net for the output probability simplex. The last step, which replaces the use of the type-counting lemma, is one of our main contributions and allows us to bound the effect of different input types with the $O(1)$ term in (2).

Note that unlike in (3), the third-order term in our upper bound in (2) is independent of $|\mathcal{X}|$. This is intuitively plausible due to the following observation. Let $n$ be a large even integer and consider using transmitting information across $n$ uses of a DMC $W: \mathcal{X} \rightarrow \mathcal{Y}$. Clearly, the same amount of information can be transmitted through $\frac{n}{2}$ uses of the product channel $W^{2}: \mathcal{X}^{\times 2} \rightarrow \mathcal{Y}^{\times 2}$, where $W^{2}\left(y, y^{\prime} \mid x, x^{\prime}\right):=W(y \mid x) W\left(y^{\prime} \mid x^{\prime}\right)$. The capacity and the dispersion of $W^{2}$ are respectively twice the capacity and the dispersion of $W$ so the normal approximation terms for $n$ uses of $W$ and $\frac{n}{2}$ uses of $W^{2}$ are identical. If the coefficient of the third-order logarithmic term were dependent on the size 
of the input alphabet, say via some function $g(|\mathcal{X}|)$, then in the first case, $\rho_{n}=g(|\mathcal{X}|) \log n+O(1)$ while in the second case, $\rho_{n}=g\left(|\mathcal{X}|^{2}\right) \log \left(\frac{n}{2}\right)+O(1)=g\left(|\mathcal{X}|^{2}\right) \log n+O(1)$. Thus, at least on an intuitive level, we expect that $g(|\mathcal{X}|)$ is independent of $|\mathcal{X}|$.

\section{Notation AND PRELiminaRies}

\section{A. Discrete Memoryless Channels}

As mentioned in the Introduction, we consider discrete memoryless channels (DMCs), which are characterized by two finite sets, the input alphabet $\mathcal{X}$ and the output alphabet $\mathcal{Y}$, and a stochastic matrix $W$, where $W(y \mid x)$ denotes the probability that the output $y \in \mathcal{Y}$ occurs given input $x \in \mathcal{X}$. The set of probability distributions on $\mathcal{X}$ is denoted $\mathcal{P}(\mathcal{X})$. For any probability distribution $P \in \mathcal{P}(\mathcal{X})$, we denote by $P \times W:(x, y) \mapsto P(x) W(y \mid x)$ the joint distribution of inputs and outputs of the channel, and by $P W: y \mapsto \sum_{x} P(x) W(y \mid x)$ its marginal on $\mathcal{Y}$. Finally, $W(\cdot \mid x)$ denotes the distribution on $\mathcal{Y}$ if the input is fixed to $x$.

Given two probability distributions $P, Q \in \mathcal{P}(\mathcal{X})$, we call the random variable $\log \frac{P(X)}{Q(X)}$ where $X$ has distribution $P$ the log-likelihood ratio of $P$ and $Q$. Its mean is the relative entropy

$$
D(P \| Q):=\mathrm{E}_{P}\left[\log \frac{P}{Q}\right]=\sum_{x \in \mathcal{X}} P(x) \log \frac{P(x)}{Q(x)}
$$

and $D(W \| Q \mid P):=\sum_{x} P(x) D(W(\cdot \mid x) \| Q)$ is the conditional information divergence. The mutual information is $I(P, W):=D(W \| P W \mid P)$. Moreover,

$$
C(W):=\max _{P \in \mathcal{P}(\mathcal{X})} I(P, W) \quad \text { and } \quad \Pi(W):=\{P \in \mathcal{P}(\mathcal{X}) \mid I(P, W)=C(W)\}
$$

are the capacity and the set of capacity-achieving input distributions (CAIDs), respectively 2 The set of CAIDs is convex and compact in $\mathcal{P}(\mathcal{X})$. The unique [13, Cor. 2 to Thm. 4.5.2] capacity-achieving output distribution (CAOD) is denoted as $Q^{*}$ and $Q^{*}=P W$ for all $P \in \Pi$. Furthermore, it satisfies $Q^{*}(y)>0$ for all $y \in \mathcal{Y}$ [13, Cor. 1 to Thm. 4.5.2], where we assume that all outputs are accessible.

The variance of the $\log$-likelihood ratio of $P$ and $Q$ is the divergence variance

$$
V(P \| Q):=\mathrm{E}_{P}\left[\left(\log \frac{P}{Q}-D(P \| Q)\right)^{2}\right] .
$$

We also define the conditional divergence variance $V(W \| Q \mid P):=\sum_{x} P(x) V(W(\cdot \mid x) \| Q)$ and the conditional information variance $V(P, W):=V(W \| P W \mid P)$. Note that $V(P, W)=V(P \times W \| P \times$ $P W)$ for all $P \in \Pi$ [4, Lem. 62]. The $\varepsilon$-channel dispersion 3 [4, Def. 2] is an operational quantity that was shown [4. Eq. (223)] to be equal to

$$
V_{\varepsilon}(W):=\left\{\begin{array}{ll}
V_{\min } & \text { if } \varepsilon<\frac{1}{2} \\
V_{\max } & \text { if } \varepsilon \geq \frac{1}{2}
\end{array}, \quad \text { where } \quad V_{\min }:=\min _{P \in \Pi} V(P, W) \quad \text { and } \quad V_{\max }:=\max _{P \in \Pi} V(P, W) .\right.
$$

Furthermore, a channel is called exotic [4, before Thm. 48] if $V_{\max }=0$ and there exists a symbol $x_{0} \in \mathcal{X}$ such that $D\left(W\left(\cdot \mid x_{0}\right) \| Q^{*}\right)=C$ and $V\left(W\left(\cdot \mid x_{0}\right) \| Q^{*}\right)>04$

For later reference, we also define the third absolute moment of the log-likelihood ratio,

$$
T(P \| Q):=\mathrm{E}_{P}\left[\left|\log \frac{P}{Q}-D(P \| Q)\right|^{3}\right]
$$

and $T(W \| Q \mid P):=\sum_{x} P(x) T(W(\cdot \mid x) \mid Q)$.

\footnotetext{
${ }^{2}$ We often drop the dependence on $W$ if it is clear from context.

${ }^{3}$ Notice that for $\varepsilon=\frac{1}{2}$, we set $V_{\varepsilon}=V_{\max }$. This is somewhat unconventional; cf. [4, Thm. 48]. However, doing so ensures that Theorem 1 can be stated compactly. Nonetheless, from the viewpoint of the normal approximation, it is immaterial how we choose $V_{\frac{1}{2}}$ since $\Phi^{-1}\left(\frac{1}{2}\right)=0$ (cf. [4, after Eq. (280)]).

${ }^{4}$ Note that this symbol must satisfy $P\left(x_{0}\right)=0$ for any $P \in \Pi$, as otherwise $V_{\max }$ would not vanish.
} 


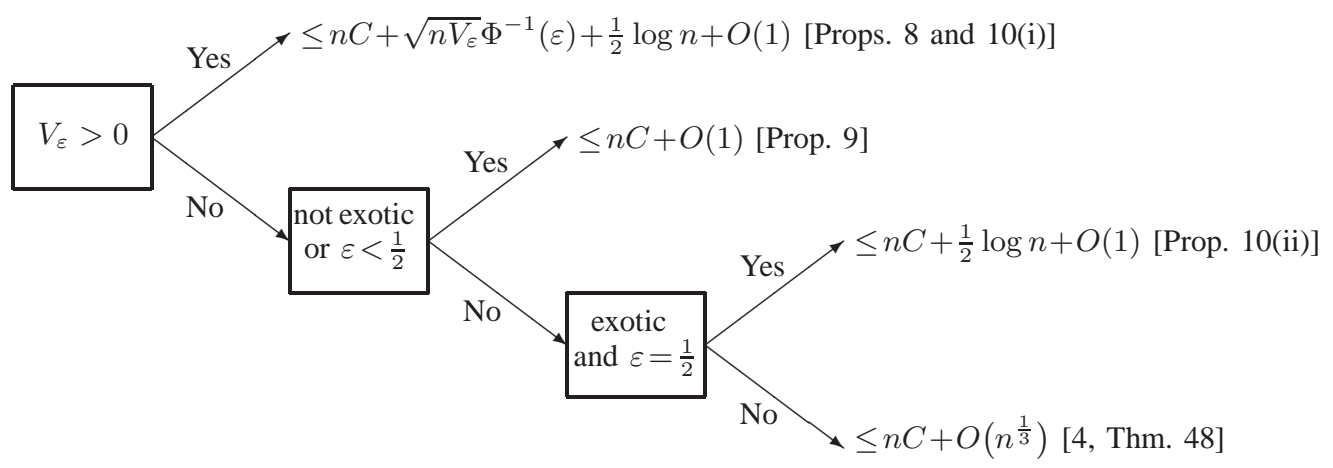

Fig. 1. Illustration of the various cases of Theorem 1 and the proof structure in Section III-E

We employ the cumulative distribution function of the standard normal distribution

$$
\Phi(a):=\int_{-\infty}^{a} \frac{1}{\sqrt{2 \pi}} \exp \left(-\frac{1}{2} x^{2}\right) \mathrm{d} x
$$

and define its inverse as $\Phi^{-1}(\varepsilon):=\sup \{a \in \mathbb{R} \mid \Phi(a) \leq \varepsilon\}$, which evaluates to the usual inverse for $0<\varepsilon<1$ and continuously extends to take values $\pm \infty$ outside that range.

For a sequence $\mathbf{x}=\left(x_{1}, x_{2}, \ldots, x_{n}\right) \in \mathcal{X}^{\times n}$, we denote by $P_{\mathbf{x}} \in \mathcal{P}(\mathcal{X})$ the probability distribution given by the relative frequencies of $\mathbf{x}$, i.e. $P_{\mathbf{x}}(x)=\frac{1}{n} \sum_{i=1}^{n} 1\left\{x_{i}=x\right\}$. This probability distribution $P_{\mathbf{x}}$ is also known as the empirical distribution or the type [11, Def. 2.1] of $\mathbf{x}$. The set of all such distributions is denoted as $\mathcal{P}_{n}(\mathcal{X})=\bigcup_{\mathbf{x}}\left\{P_{\mathbf{x}}\right\}$ and satisfies $\left|\mathcal{P}_{n}(\mathcal{X})\right| \leq(n+1)^{|\mathcal{X}|-1}$.

\section{B. Codes and $\varepsilon$-Error Capacity}

A code $\mathcal{C}$ for a channel is defined by the triple $\{\mathcal{M}, e, d\}$, where $\mathcal{M}$ is a set of messages, $e: \mathcal{M} \rightarrow \mathcal{X}$ an encoding function and $d: \mathcal{Y} \rightarrow \mathcal{M}$ a decoding function. We write $|\mathcal{C}|=|\mathcal{M}|$ for the cardinality of the message set. We define the average error probability of a code $\mathcal{C}$ for the channel $W$ as

$$
p_{\text {err }}(\mathcal{C}, W):=P\left[M \neq M^{\prime}\right]=1-\frac{1}{|\mathcal{M}|} \sum_{m \in \mathcal{M}} W\left(d^{-1}(m) \mid e(m)\right)
$$

where the distribution over messages $P_{M}$ is assumed to be uniform on $\mathcal{M}$,

$$
M \stackrel{e}{\longrightarrow} X \stackrel{W}{\longrightarrow} Y \stackrel{d}{\longrightarrow} M^{\prime}
$$

forms a Markov chain, and $M^{\prime}$ thus denotes output of the decoder. The one-shot $\varepsilon$-error capacity of the channel $W$ is then defined as

$$
M^{*}(W, \varepsilon):=\max \left\{m \in \mathbb{N}|\exists \mathcal{C}:| \mathcal{C} \mid=m \wedge p_{\text {err }}(\mathcal{C}, W) \leq \varepsilon\right\} .
$$

We are also interested in the $\varepsilon$-error capacity for $n \geq 1$ uses of a memoryless channel. For this purpose, we consider the channel $W^{n}: \mathcal{X}^{n} \rightarrow \mathcal{Y}^{n}$, defined by the stochastic matrix $W^{n}(\mathbf{y} \mid \mathbf{x})=$ $\prod_{i=1}^{n} W\left(y_{i} \mid x_{i}\right)$, where $\mathbf{x}=\left(x_{1}, x_{2}, \ldots, x_{n}\right)$ and $\mathbf{y}=\left(y_{1}, y_{2}, \ldots, y_{n}\right)$ are strings of length $n$ of symbols $x_{i} \in \mathcal{X}$ and $y_{i} \in \mathcal{Y}$, respectively. Then, the blocklength $n$, $\varepsilon$-average error capacity of the channel $W$ is denoted as $M^{*}\left(W^{n}, \varepsilon\right)$.

\section{MAIN ReSUlt AND PROOF}

Let us reiterate our main result. The various cases are illustrated diagrammatically in Fig. 1 .

Theorem 1. For every DMC $W$ and $\varepsilon$ with $V_{\varepsilon}>0$, the blocklength $n$, $\varepsilon$-error capacity satisfies

$$
\log M^{*}\left(W^{n}, \varepsilon\right) \leq n C+\sqrt{n V_{\varepsilon}} \Phi^{-1}(\varepsilon)+\frac{1}{2} \log n+O(1) .
$$


If $V_{\varepsilon}=0$, we have $\log M^{*}\left(W^{n}, \varepsilon\right) \leq n C+O(1)$, unless the channel is exotic and $\varepsilon \geq \frac{1}{2}$.

Remark 1. The $\varepsilon=\frac{1}{2}$ case needs to be treated with care. For all DMCs $W$ with $V_{\min }=0$ and $\varepsilon=\frac{1}{2}$ (this includes exotic DMCs), we show that $\log M^{*}\left(W^{n}, \varepsilon\right) \leq n C+\frac{1}{2} \log n+O(1)$. See Proposition 10 If $V_{\max }>0$, this statement concurs with the positive $\varepsilon$-dispersion case of Theorem 1

Remark 2. From the preceding statements, we see that for DMCs with $V_{\min }=0$ and $V_{\max }>0$, the third-order term "jumps" from 0 to $\frac{1}{2} \log n$ when $\varepsilon \uparrow \frac{1}{2}$. This is possible because we do not investigate the dependence of the constant term on $\varepsilon .5$

In light of the existing results on $\rho_{n}$ (in the Introduction and [5, Sec. 3.4.5]), the third-order term is the best possible unless we impose further assumptions on $W$. More precisely, it was shown in [5, Cor. 54] that if there exists a $P \in \Pi(W)$ achieving $V_{\varepsilon}(W)$ such that the reverse conditional information variance is positive, i.e. $V^{\mathrm{r}}(P, W):=V\left(P W, \frac{P \times W}{P W}\right)>0$, then

$$
\log M^{*}\left(W^{n}, \varepsilon\right) \geq n C+\sqrt{n V_{\varepsilon}} \Phi^{-1}(\varepsilon)+\frac{1}{2} \log n+O(1) .
$$

This matches the upper bound of Theorem 1

The proof consists of five parts, each detailed in one of the following subsections. In the first subsection, we introduce two entropic quantities, the hypothesis testing divergence [14]-[17] and a quantity related to the information (or divergence) spectrum [12, Ch. 4]. We state and prove some useful and well-known properties that we need later. In the second subsection, we derive a converse bound, valid for general DMCs, that involves a minimization over output distributions and maximization over input symbols. In the third subsection, we choose an appropriate output distribution for use in the general converse bound. In the fourth subsection, we state and prove some continuity properties of information measures around the CAIDs and the unique CAOD. Finally, the fifth subsection contains the proof of our main result.

\section{A. Hypothesis Testing and the Information Spectrum}

We use the following divergence [14]-[17], which is closely related to binary hypothesis testing. Let $\varepsilon \in(0,1)$ and let $P, Q \in \mathcal{P}(\mathcal{Z})$, where $\mathcal{Z}$ is finite. We consider binary (probabilistic) hypothesis tests $\xi: \mathcal{Z} \rightarrow[0,1]$ and define the $\varepsilon$-hypothesis testing divergence

$$
D_{h}^{\varepsilon}(P \| Q):=\sup \left\{R \in \mathbb{R} \mid \exists \xi: \mathrm{E}_{Q}[\xi(Z)] \leq(1-\varepsilon) \exp (-R) \wedge \mathrm{E}_{P}[\xi(Z)] \geq 1-\varepsilon\right\} .
$$

Note that $D_{h}^{\varepsilon}(P \| Q)=-\log \frac{\beta_{1-\varepsilon}(P, Q)}{1-\varepsilon}$ where $\beta_{1-\varepsilon}(P, Q)$ is the smallest type-II error of a hypothesis test between $P$ and $Q$ with type-I error smaller than $\varepsilon$ and is defined formally in [4, Eq. (100)]. It is easy to see that $D_{h}^{\varepsilon}(P \| Q) \geq 0$, where the lower bound is achieved if and only if $P=Q$ and $D_{h}^{\varepsilon}(P \| Q)$ diverges if $P$ and $Q$ are orthogonal. It satisfies a data-processing inequality [14]

$$
D_{h}^{\varepsilon}(P \| Q) \geq D_{h}^{\varepsilon}(P W \| Q W) \quad \text { for all channels } W \text { from } \mathcal{Z} \text { to } \mathcal{Z}^{\prime} .
$$

When evaluated for independent and identical distributions (i.i.d.), its asymptotic expansion in the first order is determined by the Chernoff-Stein Lemma [11, Cor. 1.2], yielding $D_{h}^{\varepsilon}\left(P^{\times n} \| Q^{\times n}\right)=$ $n D(P \| Q)+o(n)$ for any $\varepsilon \in(0,1)$. This asymptotic expansion was subsequently tightened by Juschkewitsch [18] among others. Finally Strassen [3, Thm. 3.1] found an expansion including the third-order term as

$$
D_{h}^{\varepsilon}\left(P^{\times n} \| Q^{\times n}\right)=n D(P \| Q)+\sqrt{n V(P \| Q)} \Phi^{-1}(\varepsilon)+\frac{1}{2} \log n+O(1) .
$$

\footnotetext{
${ }^{5}$ Indeed, in our proof for the case $V_{\min }=0, V_{\max }>0$ and $\varepsilon=\left(\frac{1}{2}\right)^{-}$in Proposition 9 we notice that the constant term diverges as $\varepsilon \uparrow \frac{1}{2}$.
} 
The following quantity, which characterizes the distribution of the log-likelihood ratio and is known as the relative entropy information spectrum or the divergence spectrum [12, Ch. 4], is sometimes easier to manipulate and evaluate.

$$
D_{s}^{\varepsilon}(P \| Q):=\sup \left\{R \in \mathbb{R} \mid P\left[\log \frac{P}{Q} \leq R\right] \leq \varepsilon\right\} .
$$

It is intimately related to the $\varepsilon$-hypothesis testing divergence.

Lemma 2. For any $\delta \in(0,1-\varepsilon)$, we have

$$
D_{h}^{\varepsilon}(P \| Q) \leq D_{s}^{\varepsilon+\delta}(P \| Q)+\log \frac{1-\varepsilon}{\delta} .
$$

This relation follows from standard arguments relating binary hypothesis testing and the log-likelihood test to the relative entropy information spectrum. See, for example [4, Eq. (102) and Eqs. (158)-(159)] where this is used to relax the meta-converse to (a generalization of) the Verdú-Han information spectrum converse [12, Lem. 3.2.2] or [16, Lem. 12], where an analogue of the above lemma is shown for the strictly more general non-commutative case.

We can give an upper bound on $D_{s}^{\varepsilon}(P \| Q)$ if $Q$ is a convex combination of distributions.

Lemma 3. Let $P \in \mathcal{P}(\mathcal{Z})$ and $Q=\sum_{i \in \mathcal{I}} q(i) Q^{i}$ with $Q^{i} \in \mathcal{P}(\mathcal{Z})$ and $q \in \mathcal{P}(\mathcal{I})$ and $\mathcal{I}$ is some countable index set. Then,

$$
D_{s}^{\varepsilon}(P \| Q) \leq \inf \left\{D_{s}^{\varepsilon}\left(P \| Q^{i}\right)-\log q(i)\right\}_{i \in \mathcal{I}}
$$

Proof: Note that for all $z \in \mathcal{Z}$ with $P(z)>0$, for all $i \in \mathcal{I}$, we have

$$
\log \frac{P(z)}{Q(z)}=\log \frac{P(z)}{\sum_{j} q(j) Q^{j}(z)} \leq \log \frac{P(z)}{q(i) Q^{i}(z)}=\log \frac{P(z)}{Q^{i}(z)}-\log q(i) .
$$

Hence,

$$
P\left[\log \frac{P}{Q} \leq R\right] \geq P\left[\log \frac{P}{Q^{i}} \leq R+\log q(i)\right]
$$

and thus we find $D_{s}^{\varepsilon}(P \| Q) \leq D_{s}^{\varepsilon}\left(P \| Q^{i}\right)-\log q(i)$ for any $i \in \mathcal{I}$ as desired.

The following standard result will be particularly useful, as it allows us to bound the log-likelihood ratio of the input-output behavior of two channels in terms of the log-likelihood ratio evaluated for a single input symbol.

Lemma 4. Let $P \in \mathcal{P}(\mathcal{X})$ and let $V, W$ be channels from $\mathcal{X}$ to $\mathcal{Y}$. Then,

$$
D_{s}^{\varepsilon}(P \times W \| P \times V) \leq \sup _{x: P(x)>0} D_{s}^{\varepsilon}(W(\cdot \mid x) \| V(\cdot \mid x)) .
$$

Proof: We first note that the log-likelihood ratio takes on the form

$$
\log \frac{P \times W}{P \times V}:(x, y) \mapsto \log \frac{P(x) W(y \mid x)}{P(x) V(y \mid x)}=\log \frac{W(y \mid x)}{V(y \mid x)},
$$

for every $(x, y) \in \mathcal{X} \times \mathcal{Y}$ satisfying $P(x)>0$. Now, we may write

$$
\begin{aligned}
R^{*} & =D_{s}^{\varepsilon}(P \times W \| P \times V)=\sup \left\{R \in \mathbb{R} \mid P\left[\log \frac{P \times W}{P \times V} \leq R\right] \leq \varepsilon\right\} \\
& =\sup \left\{R \in \mathbb{R} \mid \sum_{x: P(x)>0} P(x) W\left[\left\{y \mid \log \frac{W(y \mid x)}{V(y \mid x)} \leq R\right\} \mid x\right] \leq \varepsilon\right\} .
\end{aligned}
$$

Inspecting this expression, for any $\varphi>0$, we find at least one $x^{*} \in \mathcal{X}$ such that

$$
P\left(x^{*}\right)>0 \quad \text { and } \quad W\left[\left\{y \mid \log \frac{W(y \mid x)}{V(y \mid x)} \leq R\right\} \mid x\right] \leq \varepsilon .
$$


Hence, $D_{s}^{\varepsilon}\left(W\left(\cdot \mid x^{*}\right) \| V\left(\cdot \mid x^{*}\right)\right) \geq R^{*}-\varphi$, which implies the lemma as $\varphi$ is arbitrary.

The distribution of the log-likelihood ratio has the following asymptotic expansions for not necessarily identical product distributions. The bounds follow from simple applications of the Berry-Essen theorem [19, Sec. XVI.5] and Chebyshev's inequality.

Lemma 5. Let $P_{i}, Q \in \mathcal{P}(\mathcal{Z})$ be such that $Q$ dominates $P_{i}$ for all $i$ in some finite set $\mathcal{I}$. We consider a sequence of distributions $P_{i_{k}}$ indexed by $\left(i_{1}, i_{2}, \ldots, i_{n}\right)$ where $i_{k} \in \mathcal{I}$ for each $1 \leq k \leq n$. Define

$$
D_{n}:=\frac{1}{n} \sum_{k=1}^{n} D\left(P_{i_{k}} \| Q\right), \quad V_{n}:=\frac{1}{n} \sum_{k=1}^{n} V\left(P_{i_{k}} \| Q\right), \text { and } T_{n}:=\frac{1}{n} \sum_{k=1}^{n} T\left(P_{i_{k}} \| Q\right) .
$$

If $V_{n}>0$, then we have the Berry-Esseen-type bound

$$
D_{s}^{\varepsilon}\left(P_{i_{1}} \times \ldots P_{i_{n}} \| Q^{\times n}\right) \leq n D_{n}+\sqrt{n V_{n}} \Phi^{-1}\left(\varepsilon+\frac{6 T_{n}}{\sqrt{n V_{n}^{3}}}\right) .
$$

In any case, we have the Chebyshev-type bound

$$
D_{s}^{\varepsilon}\left(P_{i_{1}} \times \ldots P_{i_{n}} \| Q^{\times n}\right) \leq n D_{n}+\sqrt{\frac{n V_{n}}{1-\varepsilon}} .
$$

Proof: We consider the cumulative distribution of the random variable $S_{n}:=\sum_{k} \log P_{i_{k}}\left(X_{i_{k}}\right)-$ $\log Q\left(X_{i_{k}}\right)$ where each $X_{i_{k}}$ has distribution $P_{i_{k}}$. The random variable $S_{n}$ has mean $n D_{n}$ and variance $n V_{n}$. The general case, Eq. (5]), is shown using Chebyshev's inequality, which yields

$$
\varepsilon \geq P\left[\sum_{k} \log \frac{P_{i_{k}}}{Q} \leq R\right] \geq 1-\frac{n V_{n}}{\left(R-n D_{n}\right)^{2}} \quad \text { for } R>n D_{n}
$$

Hence, restricting to $R>n D_{n}$ and relaxing the bound on $R$ in the supremum, we find

$$
D_{s}^{\varepsilon}\left(P_{i_{1}} \times \ldots P_{i_{n}} \| Q^{\times n}\right) \leq \sup \left\{R>n D_{n} \mid 1-\frac{n V_{n}}{\left(R-n D_{n}\right)^{2}} \leq \varepsilon\right\}=n D_{n}+\sqrt{\frac{n V_{n}}{1-\varepsilon}} .
$$

Furthermore, if $V_{n}>0$, the Berry-Esseen theorem [19, Sec. XVI.5] states that

$$
\left|P\left[\sum_{k} \log \frac{P_{i_{k}}}{Q} \leq R\right]-\Phi\left(\frac{R-n D_{n}}{\sqrt{n V_{n}}}\right)\right| \leq \frac{6 T_{n}}{\sqrt{n V_{n}^{3}}} .
$$

Hence, we obtain

$$
D_{s}^{\varepsilon}\left(P_{i_{1}} \times \ldots P_{i_{n}} \| Q^{\times n}\right) \leq n D_{n}+\sqrt{n V_{n}} \Phi^{-1}\left(\varepsilon+\frac{6 T_{n}}{\sqrt{n V_{n}^{3}}}\right),
$$

which concludes the proof.

\section{B. Converse Bounds on General Channels}

Here, we give a new converse bound on the size of arbitrary codes for general channels, for the average probability of error formulation.

Proposition 6. Let $\varepsilon \in(0,1)$ and let $W$ be any channel from $\mathcal{X}$ to $\mathcal{Y}$. Then, for any $\delta \in(0,1-\varepsilon)$, we have

$$
\log M^{*}(W, \varepsilon) \leq \inf _{Q \in \mathcal{P}(\mathcal{Y})} \sup _{x \in \mathcal{X}} D_{s}^{\varepsilon+\delta}(W(\cdot \mid x) \| Q)+\log \frac{1}{\delta} .
$$

The first part of the proof is analogous to the meta-converse in [4, Thm. 27] (see also [14] and [15], which inspired our conceptually simpler proof technique). Our bound is a new "symbolwise" relaxation of the meta-converse which yields a result in the spirit of [4, Thms. 28 and 31]. 


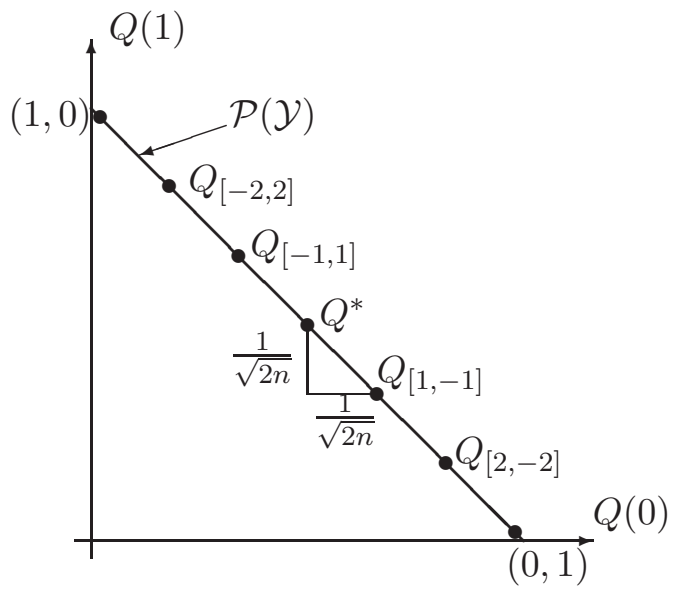

Fig. 2. Illustration of the choice of $Q_{\mathbf{k}}$ for $\mathcal{Y}=\{0,1\}$. Note that $\zeta=2$ for $|\mathcal{Y}|=2$.

The maximization over symbols allows us to apply our converse bound on non-constant-composition codes directly.

Proof: For any code $\mathcal{C}=\{\mathcal{M}, e, d\}$ with $p_{\text {err }}(\mathcal{C}) \leq \varepsilon$ and any $Q \in \mathcal{P}(\mathcal{Y})$, the following holds.

Starting from a uniform distribution over $\mathcal{M}$, the Markov chain $M \stackrel{e}{\longrightarrow} X \stackrel{W}{\longrightarrow} Y \stackrel{d}{\longrightarrow} M^{\prime}$ induces a joint probability distribution $P_{M X Y M^{\prime}}$. Due to the data-processing inequality for $D_{h}^{\varepsilon}$, we immediately find $D_{h}^{\varepsilon}(P \times W \| P \times Q)=D_{h}^{\varepsilon}\left(P_{X Y} \| P_{X} \times Q_{Y}\right) \geq D_{h}^{\varepsilon}\left(P_{M M^{\prime}} \| P_{M} \times Q_{M^{\prime}}\right)$, where $P_{X}=P$ and $Q_{M^{\prime}}$ is the distribution induced by $d$ applied to $Q_{Y}=Q 6$ Moreover, using the test $\xi\left(m, m^{\prime}\right)=$ $\delta_{m, m^{\prime}}$, we readily see that

$$
\mathrm{E}_{P \times W}\left[\xi\left(M, M^{\prime}\right)\right]=P\left[M=M^{\prime}\right] \geq 1-\varepsilon \quad \text { and } \quad \mathrm{E}_{P \times Q}\left[\xi\left(M, M^{\prime}\right)\right]=\frac{1}{|\mathcal{C}|} .
$$

Hence, $D_{h}^{\varepsilon}\left(P_{M M^{\prime}}|| P_{M} \times Q_{M^{\prime}}\right) \geq \log |\mathcal{C}|+\log (1-\varepsilon)$ by definition of the $\varepsilon$-hypothesis testing divergence. Finally, applying Lemmas 2 and 4 , we find

$$
\begin{aligned}
\sup _{x \in \mathcal{X}} D_{s}^{\varepsilon+\delta}(W(\cdot \mid x) \| Q) & \geq D_{s}^{\varepsilon+\delta}(P \times W \| P \times Q) \\
& \geq D_{h}^{\varepsilon}(P \times W \| P \times Q)-\log \frac{1-\varepsilon}{\delta} \geq \log |\mathcal{C}|-\log \frac{1}{\delta} .
\end{aligned}
$$

This yields the converse bound upon minimizing over $Q \in \mathcal{P}(\mathcal{Y})$.

\section{A Suitable Choice of Output Distribution Q}

For $n$-fold repetitions of a DMC, the bound in Proposition 6 evaluates to

$$
\log M^{*}\left(W^{n}, \varepsilon\right) \leq \min _{Q^{(n)} \in \mathcal{P}(\mathcal{Y} \times n)} \max _{\mathbf{x} \in \mathcal{X}^{\times n}} D_{s}^{\varepsilon+\delta}\left(W^{n}(\cdot \mid \mathbf{x}) \| Q^{(n)}\right)+\log \frac{1}{\delta},
$$

and it is thus important to find a suitable choice of $Q^{(n)} \in \mathcal{P}\left(\mathcal{Y}^{\times n}\right)$ to further upper bound the above. Symmetry considerations (see, e.g., [20, Sec. V]) allow us to restrict the search to distributions that are invariant under permutations of the $n$ channel uses. Let $\zeta:=|\mathcal{Y}|(|\mathcal{Y}|-1)$ and let $\gamma>0$ be a constant which is to be chosen later. Consider the following convex combination of product distributions:

$$
Q^{(n)}(\mathbf{y}):=\frac{1}{2} \sum_{\mathbf{k} \in \mathcal{K}} \frac{\exp \left(-\gamma\|\mathbf{k}\|_{2}^{2}\right)}{F} \prod_{i=1}^{n} Q_{\mathbf{k}}\left(y_{i}\right)+\frac{1}{2} \sum_{P_{\mathbf{x}} \in \mathcal{P}_{n}(\mathcal{X})} \frac{1}{\left|\mathcal{P}_{n}(\mathcal{X})\right|} \prod_{i=1}^{n} P_{\mathbf{x}} W\left(y_{i}\right),
$$

\footnotetext{
${ }^{6}$ Note that due to the Markov property, the encoding can be inverted probabilistically, without effecting the correlation between $M$ and $M^{\prime}$.
} 


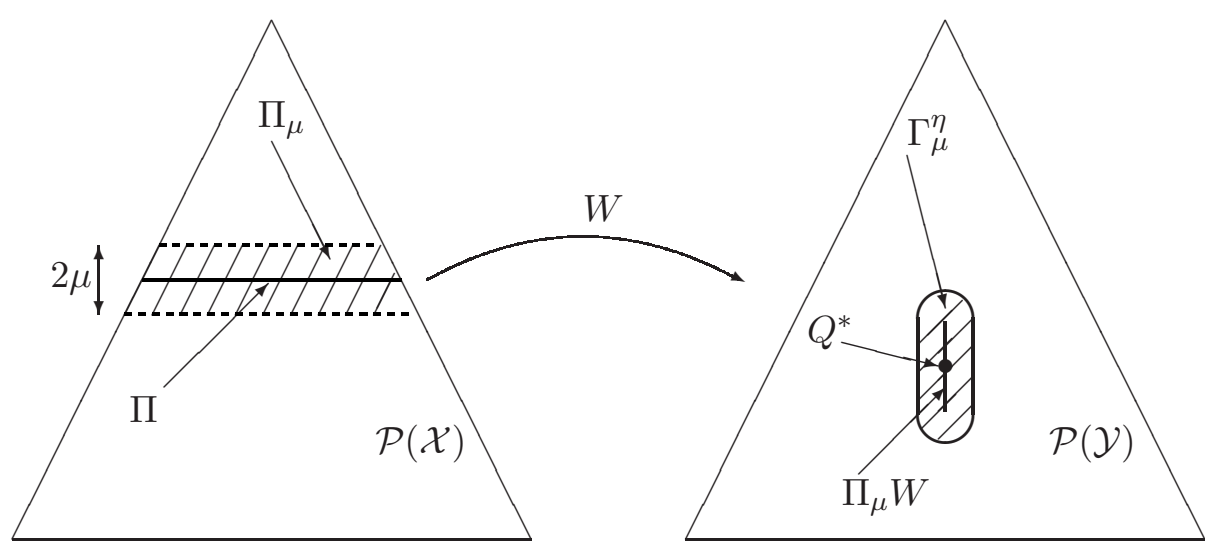

Fig. 3. Illustration of the sets in Section $\Pi$ II-D for $|\mathcal{X}|=|\mathcal{Y}|=3$. Here, $\Pi$ is not a singleton and $\Pi_{\mu} W$ has measure zero in $\mathcal{P}(\mathcal{Y})$ so $W$ is rank-deficient. The unique CAOD $Q^{*}$ is the image of $\Pi$ under $W, \Pi_{\mu} W$ is the image of $\Pi_{\mu}$ under $W$ and $\Gamma_{\mu}^{\eta}$ is the " $\eta$-blown-up" version of $\Pi_{\mu} W$.

where $F$ is a normalization constant that ensures $\sum_{\mathbf{y}} Q^{(n)}(\mathbf{y})=1$ and

$$
Q_{\mathbf{k}}(y):=Q^{*}(y)+\frac{k_{y}}{\sqrt{n \zeta}}, \quad \mathcal{K}:=\left\{\mathbf{k} \in \mathbb{Z}^{|\mathcal{Y}|} \mid \sum_{y} k_{y}=0 \wedge k_{y} \geq-Q^{*}(y) \sqrt{n \zeta}\right\} .
$$

The convex combination of $\left(P_{\mathbf{x}} W\right)^{\times n}$ and the optimal output distribution $\left(Q^{*}\right)^{\times n}$ (corresponding to $\mathbf{k}=\mathbf{0})$ in $Q^{(n)}$ is inspired partly by Hayashi [6, Thm. 2]. What we have done in our choice of $Q_{\mathbf{k}}$ is to uniformly quantize the simplex $\mathcal{P}(\mathcal{Y})$ along axis-parallel directions. The constraint that each $\mathbf{k}$ belongs to $\mathcal{K}$ ensures that each $Q_{\mathbf{k}}$ is a valid probability mass function. See Fig. 2 We find that

$$
F \leq \sum_{\mathbf{k} \in \mathbb{Z}^{|\mathcal{Y}|}} \exp \left(-\gamma\|\mathbf{k}\|_{2}^{2}\right)=\left(\sum_{k=-\infty}^{\infty} \exp \left(-\gamma k^{2}\right)\right)^{|\mathcal{Y}|} \leq\left(1+\sqrt{\frac{\pi}{\gamma}}\right)^{|\mathcal{Y}|}
$$

is a finite constant. Furthermore, by construction, the representation points $\left\{Q_{\mathbf{k}}\right\}_{\mathbf{k}}$ form an $\epsilon$-net with $\epsilon=n^{-\frac{1}{2}}$ for $\mathcal{P}(\mathcal{Y})$. Namely, for every $Q \in \mathcal{P}(\mathcal{Y})$, there exists a $\mathbf{k}$ such that $\left\|Q-Q_{\mathbf{k}}\right\|_{2} \leq n^{-\frac{1}{2}}$. This can be verified easily since by choosing a $\mathbf{k}$ that minimizes the distance in all but one direction (say the last), yielding

$$
\begin{aligned}
\left\|Q-Q_{\mathbf{k}}\right\|_{2}^{2} & =\sum_{y=1}^{|\mathcal{Y}|-1}\left(Q(y)-Q_{\mathbf{k}}(y)\right)^{2}+\left(Q(|\mathcal{Y}|)-Q_{\mathbf{k}}(|\mathcal{Y}|)\right)^{2} \\
& =\sum_{y=1}^{|\mathcal{Y}|-1}\left(Q(y)-Q_{\mathbf{k}}(y)\right)^{2}+\left(\sum_{y=1}^{|\mathcal{Y}|-1} Q_{\mathbf{k}}(y)-Q(y)\right)^{2} \\
& \leq \sum_{y=1}^{|\mathcal{Y}|-1}\left(\frac{1}{\sqrt{n \zeta}}\right)^{2}+\left(\sum_{y=1}^{|\mathcal{Y}|-1} \frac{1}{\sqrt{n \zeta}}\right)^{2}=\frac{1}{n} .
\end{aligned}
$$

Let us, at this point, provide some intuition for the choice of $Q^{(n)}$ in (6). The first part of the convex combination is used to approximate output distributions induced by inputs types that are close to the set of CAIDs. We choose a weight for each element of the $\epsilon$-net that drops exponentially with the distance from the CAOD. This ensures that the necessary normalization $F$, does not depend on $n$ even though the number of elements in the net increases with $n$. The smaller weights for types far from the CAIDs will later be compensated by the larger deviation of the corresponding mutual information from the capacity. This is achieved by the second part of the convex combination which we use to match the input types far from the CAIDs. 


\section{Continuity around the CAIDs and the unique CAOD}

We will often be concerned with probability distributions close to the set of CAIDs $\Pi$ in Euclidean distance, i.e., those distributions belonging to

$$
\Pi_{\mu}:=\left\{P \in \mathcal{P}(\mathcal{X}) \mid \min _{P^{*} \in \Pi}\left\|P-P^{*}\right\|_{2} \leq \mu\right\}
$$

for some small $\mu>0$. Sometimes we also need to restrict to probability distributions in $\Pi_{\mu}$ with positive conditional information variance. For a constant $v>0$ we define

$$
\Pi_{\mu}^{v}:=\left\{P \in \Pi_{\mu} \mid V(P, W) \geq v\right\} .
$$

The image of $\Pi_{\mu}$ under $W$ is denoted as $\Pi_{\mu} W$. We also consider a larger, " $\eta$-blown-up" version, of $\Pi_{\mu} W$, namely

$$
\Gamma_{\mu}^{\eta}:=\left\{Q \in \mathcal{P}(\mathcal{Y}) \mid \exists P \in \Pi_{\mu} \text { s.t. }\|P W-Q\|_{2} \leq \eta\right\}
$$

Note that $\Gamma_{\mu}^{0}=\Pi_{\mu} W$ if the stochastic matrix $W$ has full rank. See Fig. 3 for an illustration. The following Lemma summarizes known results about these sets.

Lemma 7. Let $W: \mathcal{X} \rightarrow \mathcal{Y}$ be a DMC and $v>0$ be a constant. There exists $\mu>0$ and $\eta>0$ and finite constants $V^{+}>0, T^{+}>0, q_{\min }>0, \alpha>0$, and $\beta>0$ such that the following holds. For all $P \in \Pi_{\mu}$ and their projections $P^{*}:=\arg \min _{P^{\prime} \in \Pi}\left\|P-P^{\prime}\right\|_{2}$ and all $Q \in \Gamma_{\mu}^{\eta}$ we have

1. $Q(y)>q_{\min }$ for all $y \in \mathcal{Y}$,

2. $V(W \| Q \mid P) \geq \frac{V_{\min }}{2}$,

3. $I(P, W) \leq C(W)-\alpha\left\|P-P^{*}\right\|_{2}^{2}$,

4. $D(W \| Q \mid P) \leq I(P, W)+\frac{\|Q-P W\|_{2}^{2}}{q_{\min }}$,

5. $V(W \| Q \mid P) \leq V^{+}$and $T(W \| Q \mid P) \leq T^{+}$.

Furthermore, for any $P \in \Pi_{\mu}^{v}$ we have

6. $V(W \| Q \mid P) \geq \frac{v}{2}>0$,

7. $\left|\sqrt{V(P, W)}-\sqrt{V\left(P^{*}, W\right)}\right| \leq \beta\left\|P-P^{*}\right\|_{2}$,

8. $|\sqrt{V(W \| Q \mid P)}-\sqrt{V(P, W)}| \leq \beta\|Q-P W\|_{2}$.

Proof: Properties 1 and 2 hold for small enough $\mu$ and $\eta$ by continuity since $Q^{*}$ has full support [13, Cor. 1 to Thm. 4.5.2] and $V\left(W \| P^{*} W \mid P^{*}\right) \geq V_{\min }$. The case $V_{\min }=0$ in Property 2 is trivial since $V(W \| Q \mid P) \geq 0$. Property 3 was established by Strassen [3, Eq. (4.41)] as well as Polyanskiy et al. [4, Eq. (501)]. Since $D(W \| Q \mid P)=I(P, W)+D(P W \| Q)$, Property 4 follows immediately from the fact that $D(P W \| Q) \leq \frac{1}{\min _{y \in \mathcal{Y}} Q(y)}\|P W-Q\|_{2}^{2}$ (see, e.g., [21, Lem. 6.3]). Property 5 follows from the fact that $(P, Q) \mapsto V(W \| Q \mid P)$ and $(P, Q) \mapsto T(W \| Q \mid P)$ are finite and continuous on the compact set $\Pi_{\mu} \times \Gamma_{\mu}^{\eta}$.

Property 6 again holds for small enough $\eta$ by continuity and since $V\left(W \| P^{*} W \mid P\right) \geq v$ by definition of the set $\Gamma_{\mu}^{\eta}$. To verify Properties 7 and 8 , note that the quotient $W(y \mid x) / Q(y)<\infty$ by Property 1. If $W(y \mid x) / Q(y)=0$, the corresponding terms in the sums defining $V(P, W)$ and $V(W \| Q \mid P)$ are excluded because $\vartheta \log ^{k} \vartheta \rightarrow 0$ as $\vartheta \rightarrow 0$ for all $k>0$. Hence, $P \mapsto V(P, W)$ and $Q \mapsto V(W \| Q \mid P)$ are continuously differentiable on $\Pi_{\mu}$ and $\Gamma_{\mu}^{\eta}$ respectively. Because $t \mapsto \sqrt{t}$ is continuously differentiable away from 0 , by Property $6, P \mapsto \sqrt{V(P, W)}$ and $Q \mapsto \sqrt{V(W \| Q \mid P)}$ are Lipschitz continuous on $\Pi_{\mu}$ and $\Gamma_{\mu}^{\eta}$ respectively. The uniformity of $\beta$ in $P$ in Property 8 can be verified by explicitly calculating the derivative of $Q \mapsto \sqrt{V(W \| Q \mid P)}$ and noting that it can be upper bounded by a finite constant independent of $P$. 


\section{E. Asymptotics for DMCs}

We are now ready to prove our main result. Several special cases of Theorem 1 require additional proof techniques. For the convenience of the reader, we state them separately as propositions. Theorem 1 then follows as a straightforward consequence of these propositions. See Fig. 1 for a summary. The following proposition considers the "regular" case, where the channel and $\varepsilon$ satisfy $V_{\varepsilon}>0$.

Proposition 8. For every DMC $W$ and $\varepsilon \in(0,1)$ such that $V_{\varepsilon}>0$, the blocklength $n$, $\varepsilon$-error capacity satisfies

$$
\log M^{*}\left(W^{n}, \varepsilon\right) \leq n C+\sqrt{n V_{\varepsilon}} \Phi^{-1}(\varepsilon)+\frac{1}{2} \log n+O(1) .
$$

Remark 3. In the following proof of Proposition 8 we deal with all cases except $\varepsilon=\frac{1}{2}, V_{\min }=0$ and $V_{\max }=V_{\varepsilon}>0$. This special case will be handled in Proposition 10 i) as it uses the proof techniques in Proposition 9

Proof: Firstly, we employ Proposition 6 to provide a bound on $\log M^{*}\left(W^{n}, \varepsilon\right)$. We choose $\delta=n^{-\frac{1}{2}}$, which satisfies $0<\delta<1-\varepsilon$ for sufficiently large $n$. Substitute the output distribution $Q^{(n)}$ in (6) to find

$$
\log M^{*}\left(W^{n}, \varepsilon\right) \leq \max _{\mathbf{x} \in \mathcal{X}^{\times n}} \underbrace{D_{s}^{\varepsilon+\delta}\left(W^{n}(\cdot \mid \mathbf{x}) \| Q^{(n)}\right)}_{=: \operatorname{cv}(\mathbf{x})}+\frac{1}{2} \log n .
$$

It remains to show that each term $\mathrm{cv}(\mathbf{x})$ in the maximization is upper bounded by $n C+\sqrt{n V_{\varepsilon}} \Phi^{-1}(\varepsilon)+$ $G$ for a suitable constant $G$ for all sufficiently large $n$.

We apply Lemma 7 which supplies us with finite, positive constants $\mu, \eta, V^{+}, T^{+}, q_{\min }, \alpha$ and $\beta$. If $V_{\min }>0$, we choose $v=\frac{V_{\min }}{2}$ such that $\Pi_{\mu}^{v}=\Pi_{\mu}$, otherwise $v>0$ will be specified later. See Case c) below.

We distinguish between three cases for the following; either a) $\mathrm{x}$ satisfies $P_{\mathbf{x}} \notin \Pi_{\mu}$ or b) $\mathbf{x}$ satisfies $P_{\mathbf{x}} \in \Pi_{\mu}^{v}$ or c) $\mathbf{x}$ satisfies $P_{\mathbf{x}} \in \Pi_{\mu} \backslash \Pi_{\mu}^{v}$. Note that Case c) is only relevant if $V_{\min }=0$, as otherwise $\Pi_{\mu}^{v}=\Pi_{\mu}$ by definition of $v$. This strategy in which we partition input types into such classes was proposed by Strassen [3, Sec. 4]. See also [4, App. I]. Intuitively, for Case a), $P_{\mathbf{x}}$ is far from the CAIDs so the first-order term is smaller than capacity; for Case $\mathrm{b}$ ), $P_{\mathbf{x}}$ has high conditional information variance and thus bounded skewness so we can apply the Berry-Esseen-type bound of Lemma 5 and; for Case c), $P_{\mathbf{x}}$ has small conditional information variance so we must use the Chebyshev-type bound and choose $v$ based on $V_{\max }$ instead of $V_{\min }$.

Case a): $P_{\mathbf{x}} \notin \Pi_{\mu}$ : The mutual information outside $\Pi_{\mu}$ is bounded away from the capacity, i.e., $I\left(P_{\mathbf{x}}, W\right) \leq C^{\prime}<C$ for all $P_{\mathbf{x}} \notin \Pi_{\mu}$.

Note that $Q^{(n)}$ can be written as a convex combination of the form in Lemma 3, where the index $i$ runs over the sets $\mathcal{K}$ and $\mathcal{P}_{n}(\mathcal{X})$. We first apply Lemma 3 to bound $\operatorname{cv}(\mathbf{x})$ with $q(i)=\frac{1}{2\left|\mathcal{P}_{n}(\mathcal{X})\right|}$ and $Q^{i}=P_{\mathbf{x}} W^{\times n}$ and then Lemma 5 to bound

$$
\begin{aligned}
\operatorname{cv}(\mathbf{x}) & \leq D_{s}^{\varepsilon+\delta}\left(W^{n}(\cdot \mid \mathbf{x}) \|\left(P_{\mathbf{x}} W\right)^{\times n}\right)+\log \left(2\left|\mathcal{P}_{n}(\mathcal{X})\right|\right) \\
& \leq n I\left(P_{\mathbf{x}}, W\right)+\sqrt{\frac{n V\left(P_{\mathbf{x}}, W\right)}{1-\varepsilon-\delta}}+\log \left(2\left|\mathcal{P}_{n}(\mathcal{X})\right|\right) .
\end{aligned}
$$

For the second inequality, we note that $D_{n}$ in Lemma 5 evaluates to

$$
D_{n}=\frac{1}{n} \sum_{i=1}^{n} \mathrm{E}_{W\left(\cdot \mid x_{i}\right)}\left[\log \frac{W\left(\cdot \mid x_{i}\right)}{P_{\mathbf{x}} W(\cdot)}\right]=\mathrm{E}_{P_{\mathbf{x}} \times W}\left[\log \frac{W}{P_{\mathbf{x}} W}\right]=D\left(W \| P_{\mathbf{x}} W \mid P_{\mathbf{x}}\right)=I\left(P_{\mathbf{x}}, W\right),
$$

and similar calculation can be done to show that $V_{n}=V\left(P_{\mathbf{x}}, W\right)$. Invoking [4, Lem. 62] and [12, Rmk. 3.1.1] yields the uniform bound $V\left(P_{\mathbf{x}}, W\right) \leq \frac{8 \log ^{2} e}{e^{2}}|\mathcal{Y}| \leq 2.3|\mathcal{Y}|$. Hence,

$$
\operatorname{cv}(\mathbf{x}) \leq n C^{\prime}+\sqrt{n} \sqrt{\frac{2.3|\mathcal{Y}|}{1-\varepsilon-\delta}}+(|\mathcal{X}|-1) \log (n+1)+\log 2 .
$$


Since $C^{\prime}<C$, the linear term dominates the term growing with the square root of $n$ and the term growing logarithmically in $n$ asymptotically. Hence, it is evident that $\operatorname{cv}(\mathbf{x}) \leq n C+\sqrt{n V_{\varepsilon}} \Phi^{-1}(\varepsilon)$ for sufficiently large $n$.

Case b): $P_{\mathbf{x}} \in \Pi_{\mu}^{v}$ : For each $\mathbf{x}$, we denote by $Q_{\mathbf{k}(\mathbf{x})}$ the element of the $\epsilon$-net (constructed in Section $\amalg I I-C)$ closest to $P_{\mathbf{x}} W$. We note that since $\left\|Q_{\mathbf{k}(\mathbf{x})}-P_{\mathbf{x}} W\right\|_{2} \leq \epsilon=n^{-\frac{1}{2}}$, we have $Q_{\mathbf{k}(\mathbf{x})} \in \Gamma_{\mu}^{\eta}$ for sufficiently large $n$, which enables us to apply the properties described in Lemma 7 extensively below.

We first use Lemma 3 with $q(i)=\frac{\exp \left(-\gamma\|\mathbf{k}(\mathbf{x})\|_{2}^{2}\right)}{2 F}$ and $Q^{i}=\left(Q_{\mathbf{k}(\mathbf{x})}\right)^{\times n}$ to bound

$$
\operatorname{cv}(\mathbf{x}) \leq D_{s}^{\varepsilon+\delta}\left(W^{n}(\cdot \mid \mathbf{x}) \|\left(Q_{\mathbf{k}(\mathbf{x})}\right)^{\times n}\right)+\gamma\|\mathbf{k}(\mathbf{x})\|_{2}^{2}+\log (2 F) .
$$

We now employ Lemma 5, where we choose $P_{i}=W\left(\cdot \mid x_{i}\right)$ resulting in $D_{n}:=D\left(W \| Q_{\mathbf{k}(\mathbf{x})} \mid P_{\mathbf{x}}\right)$, $V_{n}:=V\left(W \| Q_{\mathbf{k}(\mathbf{x})} \mid P_{\mathbf{x}}\right)$ and $T_{n}:=T\left(W \| Q_{\mathbf{k}(\mathbf{x})} \mid P_{\mathbf{x}}\right)$. From Lemma 7 we have that $T_{n} \leq T^{+}$and $0<\frac{v}{2}<V_{n} \leq V^{+}$. We then introduce the finite constant $B:=1+6 \sqrt{8} T_{+} / v^{\frac{3}{2}}$, while substituting for $\delta=n^{-\frac{1}{2}}$, to find

$$
\operatorname{cv}(\mathbf{x}) \leq n D\left(W \| Q_{\mathbf{k}(\mathbf{x})} \mid P_{\mathbf{x}}\right)+\sqrt{n V\left(W \| Q_{\mathbf{k}(\mathbf{x})} \mid P_{\mathbf{x}}\right)} \Phi^{-1}\left(\varepsilon+\frac{B}{\sqrt{n}}\right)+\gamma\|\mathbf{k}(\mathbf{x})\|_{2}^{2}+\log (2 F) .
$$

We now require that $n \geq N$, where $N$ is chosen large enough such that $\varepsilon+\frac{B}{\sqrt{N}}<1$. This ensures that the coefficient of the term growing as $\sqrt{n}$ in the above expression is finite. Next, we use the fact that $\Phi^{-1}$ is infinitely differentiable and $V\left(W \| Q_{\mathbf{k}(\mathbf{x})} \mid P_{\mathbf{x}}\right) \leq V_{+}$is finite to bound

$$
\sqrt{n V\left(W \| Q_{\mathbf{k}(\mathbf{x})} \mid P_{\mathbf{x}}\right)} \Phi^{-1}\left(\varepsilon+\frac{B}{\sqrt{n}}\right) \leq \sqrt{n V\left(W \| Q_{\mathbf{k}(\mathbf{x})} \mid P_{\mathbf{x}}\right)} \Phi^{-1}(\varepsilon)+G_{1} .
$$

for some finite constant $G_{1}$ and all $n \geq N$. Thus, defining $G_{2}:=G_{1}+\log (2 F)$, we find

$$
\operatorname{cv}(\mathbf{x}) \leq n D\left(W \| Q_{\mathbf{k}(\mathbf{x})} \mid P_{\mathbf{x}}\right)+\sqrt{n V\left(W \| Q_{\mathbf{k}(\mathbf{x})} \mid P_{\mathbf{x}}\right)} \Phi^{-1}(\varepsilon)+\gamma\|\mathbf{k}(\mathbf{x})\|_{2}^{2}+G_{2},
$$

Next, we would like to replace $Q_{\mathbf{k}(\mathbf{x})}$ with $P_{\mathbf{x}} W$ in the above bound. This can be done without too much loss due to Lemma 7 , which states that

and

$$
D\left(W \| Q_{\mathbf{k}(\mathbf{x})} \mid P_{\mathbf{x}}\right) \leq I\left(P_{\mathbf{x}}, W\right)+\frac{\left\|P_{\mathbf{x}} W-Q_{\mathbf{k}(\mathbf{x})}\right\|_{2}^{2}}{q_{\min }} \leq I\left(P_{\mathbf{x}}, W\right)+\frac{1}{n q_{\min }}
$$

$$
\left|\sqrt{V\left(W \| Q_{\mathbf{k}(\mathbf{x})} \mid P_{\mathbf{x}}\right)}-\sqrt{V\left(P_{\mathbf{x}}, W\right)}\right| \leq \beta\left\|P_{\mathbf{x}} W-Q_{\mathbf{k}(\mathbf{x})}\right\|_{2} \leq \frac{\beta}{\sqrt{n}} .
$$

Hence, choosing $G_{3}:=\frac{1}{q_{\min }}+\beta\left|\Phi^{-1}(\varepsilon)\right|+G_{2}$, we find that

$$
\operatorname{cv}(\mathbf{x}) \leq n I\left(P_{\mathbf{x}}, W\right)+\sqrt{n V\left(P_{\mathbf{x}}, W\right)} \Phi^{-1}(\varepsilon)+\gamma\|\mathbf{k}(\mathbf{x})\|_{2}^{2}+G_{3} .
$$

In the following, we use the fact that all distributions (and types) $P_{\mathbf{x}}$ in $\Pi_{\mu}$ satisfy $I\left(P_{\mathbf{x}}, W\right) \leq$ $C-\alpha \xi^{2}$ and $\left|\sqrt{V\left(P_{\mathbf{x}}, W\right)}-\sqrt{V\left(P^{*}, W\right)}\right| \leq \beta \xi$, where $P^{*}:=\arg \min _{P^{\prime} \in \Pi}\left\|P_{\mathbf{x}}-P^{\prime}\right\|_{2}$ (which is unique) and $\xi:=\left\|P_{\mathbf{x}}-P^{*}\right\|_{2}$. Hence,

$$
\mathrm{cv}(\mathbf{x}) \leq n C+\sqrt{n V\left(P^{*}, W\right)} \Phi^{-1}(\varepsilon)+\left(-\alpha \xi^{2} n+\beta\left|\Phi^{-1}(\varepsilon)\right| \xi \sqrt{n}+\gamma\|\mathbf{k}(\mathbf{x})\|_{2}^{2}\right)+G_{3} .
$$

It thus remains to show that the term in the bracket is upper bounded by a constant, for an appropriate choice of $\gamma$. Let $\|W\|_{2}:=\max \left\{\|\mathbf{u} W\|_{2} \mid\|\mathbf{u}\|_{2} \leq 1\right\}$ be the spectral norm of the matrix $W$. It is easy to see that $\|W\|_{2} \leq \sqrt{|\mathcal{X}|}$. From the construction of the $\epsilon$-net in Section III-C

$$
\begin{aligned}
\|\mathbf{k}(\mathbf{x})\|_{2} & =\sqrt{n \zeta}\left\|Q_{\mathbf{k}(\mathbf{x})}-Q^{*}\right\|_{2} \\
& \leq \sqrt{n \zeta}\left(\left\|Q_{\mathbf{k}(\mathbf{x})}-P_{\mathbf{x}} W\right\|_{2}+\left\|P_{\mathbf{x}} W-Q^{*}\right\|_{2}\right) \\
& \leq \sqrt{n \zeta}\left(\frac{1}{\sqrt{n}}+\|W\|_{2} \xi\right) .
\end{aligned}
$$


Substituting this bound into (7), we find that the term in the bracket evaluates to

$$
\left(\gamma \zeta\|W\|_{2}^{2}-\alpha\right) \xi^{2} n+\left(\beta\left|\Phi^{-1}(\varepsilon)\right|+2 \gamma \zeta\|W\|_{2}\right) \xi \sqrt{n}+\gamma \zeta
$$

The expression is a quadratic polynomial in $\xi \sqrt{n}$ and has a finite maximum if we choose $\gamma$ such that $\gamma \zeta\|W\|_{2}^{2}<\alpha$. (Note that $\|W\|_{2}>0$ for any channel.) Hence, we can write

$$
\operatorname{cv}(\mathbf{x}) \leq n C+\sqrt{n V\left(P^{*}, W\right)} \Phi^{-1}(\varepsilon)+G_{4}
$$

for an appropriate constant $G_{4}$ and $n \geq N$.

Case c) $P_{\mathbf{x}} \in \Pi_{\mu} \backslash \Pi_{\mu}^{v}$ : Note that this case only appears if $V_{\min }=0, V_{\max }=V_{\varepsilon}>0$ and $\varepsilon \geq \frac{1}{2}$. We consider the case $\varepsilon>\frac{1}{2}$ (cf. Remark 3) leaving the $\varepsilon=\frac{1}{2}$ case for Proposition 10 (i). We have

$$
\begin{aligned}
\operatorname{cv}(\mathbf{x}) & \leq D_{s}^{\varepsilon+\delta}\left(W^{n}(\cdot \mid \mathbf{x}) \|\left(P_{\mathbf{x}} W\right)^{\times n}\right)+\log \left(2\left|\mathcal{P}_{n}(\mathcal{X})\right|\right) \\
& \leq n I\left(P_{\mathbf{x}}, W\right)+\sqrt{\frac{n V\left(P_{\mathbf{x}}, W\right)}{1-\varepsilon-\delta}}+\log \left(2\left|\mathcal{P}_{n}(\mathcal{X})\right|\right) \\
& \leq n I\left(P_{\mathbf{x}}, W\right)+\sqrt{\frac{n v}{1-\varepsilon-\delta}}+\log \left(2\left|\mathcal{P}_{n}(\mathcal{X})\right|\right)
\end{aligned}
$$

Now we choose $v>0$ to be any constant satisfying

$$
\sqrt{\frac{v}{1-\varepsilon-\delta}}+\frac{\log \left(2\left|\mathcal{P}_{n}(\mathcal{X})\right|\right)}{\sqrt{n}} \leq \sqrt{V_{\max }} \Phi^{-1}(\varepsilon) .
$$

It is certainly possible to find such a $v$ since the number of types is polynomial so $\delta$ and the second term on the left are arbitrarily small for large enough $n$. Furthermore, $\sqrt{V_{\max }} \Phi^{-1}(\varepsilon)>0$. This is where $\varepsilon \neq \frac{1}{2}$ is crucial. Uniting the preceding two bounds yields

$$
\mathrm{cv}(\mathbf{x}) \leq n I\left(P_{\mathbf{x}}, W\right)+\sqrt{n V_{\max }} \Phi^{-1}(\varepsilon) \leq n C+\sqrt{n V_{\max }} \Phi^{-1}(\varepsilon) .
$$

Summarizing the bounds for Cases a), b) and c), we thus have the following asymptotic expansion for all $n$ sufficiently large:

$$
\begin{aligned}
\log M^{*}\left(W^{n}, \varepsilon\right) & \leq \max _{P^{*} \in \Pi} n C+\sqrt{n V\left(P^{*}, W\right)} \Phi^{-1}(\varepsilon)+\frac{1}{2} \log n+G_{4} \\
& =n C+\sqrt{n V_{\varepsilon}} \Phi^{-1}(\varepsilon)+\frac{1}{2} \log n+G_{4},
\end{aligned}
$$

where the last equality follows by definition of $V_{\varepsilon}$.

Surprisingly, the first-order approximation is accurate up to a constant term if $V_{\varepsilon}=0$ unless the channel is exotic and $\varepsilon \geq \frac{1}{2}$.

Proposition 9. For every DMC $W$ and $\varepsilon \in(0,1)$ such that $V_{\varepsilon}=0$, the blocklength $n$, $\varepsilon$-error capacity satisfies $\log M^{*}\left(W^{n}, \varepsilon\right) \leq n C+O(1)$, unless the channel is exotic and $\varepsilon \geq \frac{1}{2}$.

Proof: Again, from our bound on the converse for general channels (Proposition 6), we have

$$
\log M^{*}\left(W^{n}, \varepsilon\right) \leq \max _{\mathbf{x} \in \mathcal{X}^{\times n}} \underbrace{D_{s}^{\varepsilon+\delta}\left(W^{n}(\cdot \mid \mathbf{x}) \| Q^{(n)}\right)}_{=: \operatorname{cv}(\mathbf{x})}+\log \frac{1}{\delta} .
$$

We upper bound $\mathrm{cv}(\mathbf{x})$ using Lemma 3 (picking out the $\mathbf{k}=\mathbf{0}$ term) as follows:

$$
\operatorname{cv}(\mathbf{x}) \leq D_{s}^{\varepsilon+\delta}\left(W^{n}(\cdot \mid \mathbf{x}) \|\left(Q^{*}\right)^{\times n}\right)+\log (2 F) .
$$

We also choose $\delta=\frac{1}{2}-\varepsilon$ if $\varepsilon<\frac{1}{2}$ and $\delta=\frac{1-\varepsilon}{2}$ otherwise; hence, the term $\log \frac{1}{\delta}$ is finite and independent of $n$. Also let $m(\mathbf{x})$ be the number of non-zero variance letters in x, i.e., $m(\mathbf{x}):=$ $n P_{\mathbf{x}}\left(\mathcal{X}_{+}\right)=\sum_{i=1}^{n} 1\left\{x_{i} \in \mathcal{X}_{+}\right\}$where $\mathcal{X}_{+}:=\left\{x \in \mathcal{X}: V\left(W(\cdot \mid x) \| Q^{*}\right)>0\right\}$. There exist finite constants $v_{\min }, v_{\max }$ and $t_{\max }$ such that, for every $x \in \mathcal{X}_{+}$,

$$
0<v_{\min } \leq V\left(W(\cdot \mid x) \| Q^{*}\right) \leq v_{\max }, \quad \text { and } \quad T\left(W(\cdot \mid x) \| Q^{*}\right) \leq t_{\max } .
$$


By the definitions of $D_{n}:=D\left(W \| Q^{*} \mid P_{\mathbf{x}}\right), V_{n}:=V\left(W \| Q^{*} \mid P_{\mathbf{x}}\right)$ and $T_{n}:=T\left(W \| Q^{*} \mid P_{\mathbf{x}}\right)(\mathrm{cf}$. Lemma[5), we have

$$
\frac{m(\mathbf{x})}{n} v_{\min } \leq V_{n} \leq \frac{m(\mathbf{x})}{n} v_{\max }, \quad \text { and } \quad T_{n} \leq \frac{m(\mathbf{x})}{n} t_{\max }
$$

Further defining $B_{n}:=6 T_{n} / V_{n}^{\frac{3}{2}}$, we thus find

$$
B_{n} \leq \sqrt{\frac{n}{m(\mathbf{x})}} L \quad \text { where } \quad L:=\frac{6 t_{\max }}{v_{\min }^{3 / 2}}<\infty .
$$

Let $m^{*}$ be an integer satisfying $L / \sqrt{m^{*}} \leq r^{\prime}$ where $r^{\prime}$ is chosen such that $\Phi^{-1}\left(\frac{1}{2}+r\right) \leq 3 r$ for all $r \in\left[0, r^{\prime}\right]$. The choice $r^{\prime}=0.35$ does the job.

For $\varepsilon<\frac{1}{2}$, following Strassen's argument [3, Eq. (4.53)-(4.54)] (see also [4, App. I]), we distinguish between two classes of sequences as follows: the sequence $\mathbf{x}$ satisfies either a) $m(\mathbf{x}) \geq m^{*}$, or $\mathbf{b}$ ) $m(\mathbf{x})<m^{*}$. Finally, c) considers the case where $W$ is not exotic and $\varepsilon \geq \frac{1}{2}$. Intuitively, for Case a), we can use the Berry-Esseen-type bound because $m(\mathbf{x})$ is large, and hence $B_{n}$ can be bounded appropriately; for Case $\mathbf{b}$ ), we use the Chebyshev-type bound because $m(\mathbf{x})$ is small and; for Case c), we use the non-exoticness of $W$ to bound $D_{n}$ far away from $C$.

Case a): $\varepsilon<\frac{1}{2}$ and $m(\mathbf{x}) \geq m^{*}$ : We apply the Berry-Esseen-type bound in Lemma 5 to (8) to find

$$
\begin{aligned}
\operatorname{cv}(\mathbf{x}) & \leq n D_{n}+\sqrt{n V_{n}} \Phi^{-1}\left(\varepsilon+\delta+\frac{B_{n}}{\sqrt{n}}\right) \\
& \leq n D_{n}+\sqrt{n V_{n}} \Phi^{-1}\left(\frac{1}{2}+\frac{L}{\sqrt{m(\mathbf{x})}}\right) \leq n D_{n}+3 L \sqrt{\frac{n V_{n}}{m(\mathbf{x})}} .
\end{aligned}
$$

Here, we used the fact that $\varepsilon+\delta=\frac{1}{2}$ by definition of $\delta$ and the proof concludes with the observation that $\frac{n V_{n}}{m(\mathbf{x})} \leq v_{\max }$ is bounded by a constant, and $D_{n} \leq C$ for all $\mathbf{x}$.

Case $b$ ): $\varepsilon<\frac{1}{2}$ and $m(\mathbf{x})<m^{*}$ : We use the Chebyshev-type bound in Lemma 5 to (8) yielding

$$
\operatorname{cv}(\mathbf{x}) \leq n D_{n}+\sqrt{\frac{n V_{n}}{1-\varepsilon-\delta}}=n D_{n}+\sqrt{2 n V_{n}} .
$$

Since by (9), $n V_{n} \leq m^{*} v_{\max }$ and $D_{n} \leq C$ for all $\mathrm{x}$, we find the desired bound.

Case c): not exotic, $\varepsilon \geq \frac{1}{2}$ : Lemma 5 applied to (8) again yields

$$
\mathrm{cv}(\mathbf{x}) \leq n D_{n}+\sqrt{\frac{n V_{n}}{1-\varepsilon-\delta}}=n D_{n}+\sqrt{\frac{2 n V_{n}}{1-\varepsilon}},
$$

because in this case, $\delta=\frac{1-\varepsilon}{2}$. By virtue of the fact that $V_{\max }=0$ and $W$ is not exotic, we have that either

$$
D\left(W(\cdot \mid x) \| Q^{*}\right)<C \quad \text { or } \quad V\left(W(\cdot \mid x) \| Q^{*}\right)=0
$$

for all symbols $x \in \mathcal{X}$. If $\mathcal{X}_{+}$is empty, we have $V_{n}=0$ and the bound is immediate. Otherwise, we define $\psi:=C-\max _{x \in \mathcal{X}_{+}} D\left(W(\cdot \mid x) \| Q^{*}\right)>0$, which is positive due to the condition in (12).

Using this, we find that $n D_{n} \leq n C-m(\mathbf{x}) \psi$ and $n V_{n} \leq v_{\max } m(\mathbf{x})$ by 91. Thus,

$$
\mathrm{cv}(\mathbf{x}) \leq n C-m(\mathbf{x}) \psi+\sqrt{\frac{2 m(\mathbf{x}) v_{\max }}{1-\varepsilon}}
$$

The latter two terms constitute a quadratic polynomial in $\sqrt{m(\mathbf{x})}$, and hence, their sum has a finite maximum.

Finally, we deal with the case that was left out in Proposition 8

Proposition 10. Let $\varepsilon=\frac{1}{2}$. The following hold: 
(i) For every DMC $W$ such that $V_{\min }=0$ and $V_{\max }>0$, the blocklength $n$, $\varepsilon$-error capacity satisfies $\log M^{*}\left(W^{n}, \varepsilon\right) \leq n C+\frac{1}{2} \log n+O(1)$.

(ii) For every exotic DMC $W$ (in particular, $V_{\max }=0$ ), the same bound as in (i) holds.

Proof: By placing no assumptions on $V_{\max } \geq 0$, we can prove both parts in tandem. The proof follows closely that of Proposition 9 with the exception that we choose $\delta=n^{-\frac{1}{2}}$ so the $\log \frac{1}{\delta}$ term evaluates to $\frac{1}{2} \log n$. It remains to show that $\mathrm{cv}(\mathbf{x}) \leq n C+O(1)$. We split the analysis into Cases a) and b) as in Proposition 9 and let $D_{n}:=D\left(W \| Q^{*} \mid P_{\mathbf{x}}\right)$ and $V_{n}:=V\left(W \| Q^{*} \mid P_{\mathbf{x}}\right)$.

Case $a$ ): $\varepsilon=\frac{1}{2}, V_{\min }=0$ and $m(\mathbf{x}) \geq m^{*}:$ By the same steps that led to (10), we have

$$
\operatorname{cv}(\mathbf{x}) \leq n D_{n}+3(L+1) \sqrt{\frac{n V_{n}}{m(\mathbf{x})}}
$$

because $\delta=n^{-\frac{1}{2}}$. We obtain the desired bound by noting that $\frac{n V_{n}}{m(\mathbf{x})} \leq v_{\max }$ and $D_{n} \leq C$.

Case $b$ ): $\varepsilon=\frac{1}{2}, V_{\min }=0$ and $m(\mathbf{x})<m^{*}:$ By the same steps that led to (11), we have

$$
\operatorname{cv}(\mathbf{x}) \leq n D_{n}+\sqrt{4 n V_{n}}
$$

because $1-\varepsilon-\delta=\frac{1}{2}-\delta \geq \frac{1}{4}$ for all $n \geq 4$. The proof is completed by noting that $n V_{n} \leq m^{*} v_{\max }$ and $D_{n} \leq C$.

Proof of Theorem [1: The first statement follows by Propositions 8 and 10(i). The second statement follows by Proposition 9

\section{Conclusion And Open Problems}

We have presented improved converse (upper) bounds on the blocklength $n, \varepsilon$-average error capacity $M^{*}\left(W^{n}, \varepsilon\right)$. These bounds are tight in the third-order for all DMCs with positive reverse dispersion [5, Thm. 53]. However, the BEC (with zero reverse dispersion) is a notable example for which our result is not tight and in fact overestimates $\log M^{*}\left(W^{n}, \varepsilon\right)$ by $\frac{1}{2} \log n$. To prove a tight converse bound on the third-order for the BEC, a different non-product choice for $Q^{(n)}$ is necessary, as was pointed out recently by Polyanskiy [20, Thm. 23]. It remains to investigate whether a combination of Polyanskiy's choice and our choice of output distribution can be used to derive tight third-order asymptotic bounds for all DMCs.

Our general converse bound in Proposition 6 can be specialized to channels with cost constraints. As such, it can be applied to the AWGN channel with maximal (or equal) power constraints and the evaluation of Proposition 6 using the product CAOD yields the $\frac{1}{2} \log n+O(1)$ upper bound on the third-order term [4, Thm. 54]. It would be interesting to check if the evaluation of Proposition 6 yields the same upper bound for the finite-dimensional infinite constellations problem [22, Thm. 13].

Acknowledgements: MT thanks Ligong Wang for helpful explanations. VYFT thanks Yanina Shkel for insightful discussions and Pierre Moulin for sharing his ITA paper [10]. MT is supported by the National Research Foundation and the Ministry of Education of Singapore. VYFT would like to acknowledge funding support from the Agency for Science, Technology and Research (A*STAR), Singapore.

\section{REFERENCES}

[1] C. E. Shannon. A mathematical theory of communication. Bell System Tech. Journal, 27:379-423, 1948.

[2] J. Wolfowitz. Coding Theorems of Information Theory. Springer-Verlag, New York, 3rd edition, 1978.

[3] V. Strassen. Asymptotische Abschätzungen in Shannons Informationstheorie. In Trans. Third Prague Conf. Inf. Theory, pages 689-723, Prague, 1962.

[4] Y. Polyanskiy, H. V. Poor, and S. Verdú. Channel coding in the finite blocklength regime. IEEE Trans. on Inf. Th., 56:2307-59, May 2010.

[5] Y. Polyanskiy. Channel coding: Non-asymptotic fundamental limits. PhD thesis, Princeton University, 2010.

[6] M. Hayashi. Information spectrum approach to second-order coding rate in channel coding. IEEE Trans. on Inf. Th., 55:4947-66, Nov 2009. 
[7] P. Moulin. The log-volume of optimal constant-composition codes for memoryless channels, within O(1) bits. In Int. Symp. Inf. Th., Cambridge, MA, 2012.

[8] N. R. Chaganty and J. Sethuraman. Strong large deviation and local limit theorems. Ann. Prob., 21(3):1671-90, 1993.

[9] R. Bahadur and R. Ranga. On deviations of the sample mean. Ann. Math. Stat., 31:1015-27, 1960.

[10] P. Moulin. The log-volume of optimal codes for memoryless channels, up to a few nats. In Info. Th. and Appl. Workshop, San Diego, CA, 2012.

[11] I. Csiszár and J. Körner. Information Theory: Coding Theorems for Discrete Memoryless Systems. Cambridge University Press, 2011.

[12] T. S. Han. Information-Spectrum Methods in Information Theory. Springer Berlin Heidelberg, Feb 2003.

[13] R. G. Gallager. Information Theory and Reliable Communication. Wiley, New York, 1968.

[14] L. Wang, R. Colbeck, and R. Renner. Simple channel coding bounds. In Intl. Symp. Inf. Th., Seoul, South Korea, 2009.

[15] L. Wang and R. Renner. One-shot classical-quantum capacity and hypothesis testing. Physical Review Letters, 108:200501, May 2012.

[16] M. Tomamichel and M. Hayashi. A hierarchy of information quantities for finite block length analysis of quantum tasks. arXiv: 1208.1478 [quant-ph], Sep 2012.

[17] F. Dupuis, L. Krämer, P. Faist, J. M. Renes, and R. Renner. Generalized entropies. arXiv:1211.3141 [quant-ph], Nov 2012.

[18] Juschkewtisch. Über einen Grenzwertsatz, der mit dem Begriff der Entropie einer Markoffschen Kette zusammenhängt. Uspechi Mat. Nauk VIII, 5, 1953.

[19] W. Feller. An Introduction to Probability Theory and Its Applications. John Wiley and Sons, 2nd edition, 1971.

[20] Y. Polyanskiy. Saddle point in the minimax converse for channel coding. IEEE Trans. on Inf. Theory, 59(5):2576-95, May 2013.

[21] I. Csiszár and Z. Talata. Context tree estimation for not necessarily finite memory processes, via BIC and MDL. IEEE Trans. on Inf. Th., 52(3):1007-16, Mar 2006.

[22] A. Ingber, R. Zamir, and M. Feder. Finite-dimensional infinite constellations. IEEE Trans. on Inf. Th., 59(3):1630-56, 2013. 